$\underline{R E V I E W}$

\title{
A role for the nasal cycle in respiratory defence
}

\author{
R. Eccles
}

A role for the nasal cycle in respiratory defence. R. Eccles. (OERS Journals Ltd 1996. ABSTRACT: This review describes the phenomenon of the nasal cycle, which consists of periodic congestion and decongestion of the nasal venous sinusoids.

The hypothesis is put forward that the nasal venous sinusoids participate in respiratory defence by generation of plasma exudate. This hypothesis is based on recent studies, which have shown that the nasal venous sinusoids have a fenestrated endothelium and that the nasal cycle is increased during periods of nasal infection; and also on a series of older observations in the literature, which link the generation of nasal fluid to the decongestion of nasal venous sinusoids.

It is proposed that the periodic congestion and decongestion of nasal venous sinusoids may provide a pump mechanism for the generation of plasma exudate, and that this mechanism is an important component of respiratory defence.

Eur Respir J., 1996, 9, 371-376.
Common Cold and Nasal Research Centre, University of Wales College of Cardiff, Cardiff, UK.

Correspondence: R. Eccles, Common Cold and Nasal Research Centre, 56 Park Place, University of Wales College of Cardiff, Cardiff CF1 3AT, UK.

Keywords: Nasal airway resistance, nasal cycle, nose, respiratory defence

Received: June 91995

Accepted after revision November 51995
The airflow through the nasal passage is normally asymmetrical, with one nasal passage having the dominant airflow. This asymmetry of nasal airflow is not fixed, as the dominant airflow alternates from one nasal passage to the other over a period of several hours [1-3]. The asymmetry in nasal airflow is exaggerated with nasal infection and one nasal passage may become completely obstructed whilst the other remains patent $[4,5]$. The spontaneous reciprocal changes in nasal airway resistance are often described as the "nasal cycle".

The functional significance of the nasal cycle is obscure and textbooks on the nose offer no real explanation for this unusual phenomenon [6-9].

Recent studies on the generation of nasal plasma exudate as a first line of respiratory defence by PERSSON and co-workers [10-13], together with some elegant anatomical studies on the structure of nasal blood vessels by GrEvers and co-workers [14-20], and reports that the amplitude of the nasal cycle is increased during nasal infection $[4,5]$, have provided new knowledge, which may help explain the functional basis of the nasal cycle.

The present review will integrate this new knowledge into previously reported information about the nasal cycle and put forward the hypothesis that the periodic congestion and decongestion of the nasal mucosa associated with the nasal cycle is an important component of respiratory defence.

\section{Nasal cycle}

The airflow through the two nasal passages is normally asymmetrical and this asymmetry is exaggerated with viral infection of the nose $[4,5]$, and on adoption of a supine posture [21]. The dominance in nasal airflow alternates from one passage to the other over a period of several hours and this phenomenon is often referred to as the "nasal cycle". Examples of spontaneous reciprocal changes in nasal airway resistance in one subject in health and disease are illustrated in figure 1, which demonstrates the increased amplitude of the changes in unilateral nasal resistance associated with symptoms of acute upper respiratory tract infection [5]. The changes in nasal resistance associated with the nasal cycle are not always as regular as those illustrated in figure 1, and the term nasal cycle may be a misnomer, as there is little evidence to indicate a regular periodicity to the changes in nasal resistance. The changes in nasal resistance are not always reciprocal and some subjects may exhibit changes in nasal resistance which are in phase or appear to have no relationship at all between the two sides of the nose. Some patients with nasal infection may complain of bilateral nasal obstruction, especially if there is a deviated nasal septum, but this type of complete nasal obstruction was not found in the study by ECCLES et al. [5], or in the study by BENDE et al. [4].

One of the first studies on the nasal cycle was reported in 1895 by KAYSER [22], who measured the resistance of the nose by timing the period required to withdraw a definite volume of air through the nose via the oral cavity. Kayser found consistent measurements for total nasal resistance but found that the resistance of the separate nasal passages greatly fluctuated. On the basis of his findings, KAYSER [22] established that the nasal passages are subject to continual rhythmic changes in nasal resistance. The reciprocal spontaneous changes in unilateral nasal airway resistance, which constitute a nasal cycle, have been well documented by subsequent investigators [1-3, 23-29]. The term "nasal cycle" is perhaps misleading as the spontaneous reciprocal changes in nasal 

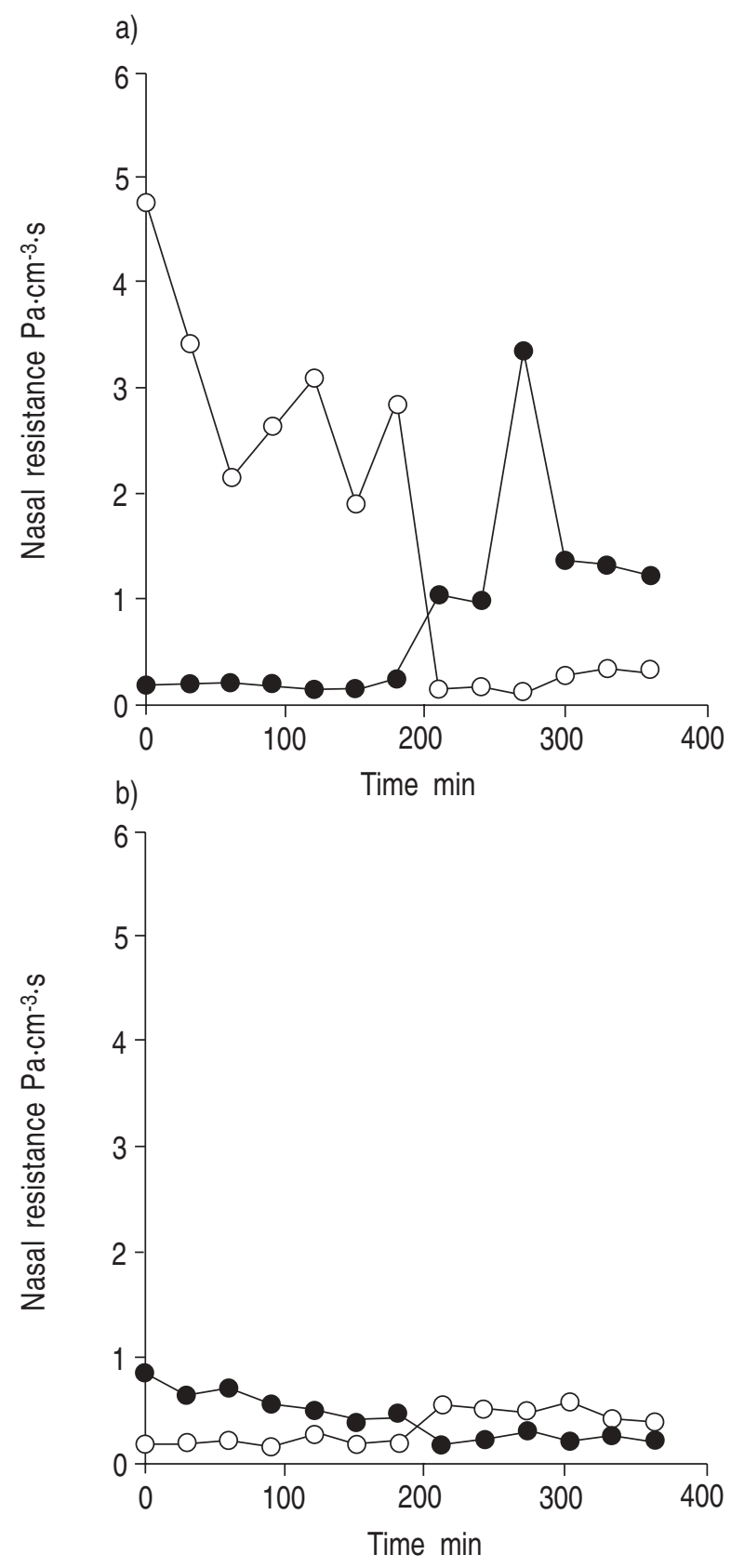

Fig. 1. - Spontaneous reciprocal changes in unilateral nasal airway resistance recorded in one subject: a) whilst suffering from symptoms of acute upper respiratory tract infection; and b) 6-8 weeks later when healthy. $\mathrm{O}$ : left nasal resistance; resistance. Each point represents the mean nasal airway resistance calculated from 12 breaths. (Redrawn from EcCles et al. [5] 1995).

airway resistance have only rarely been shown to exhibit any regular pattern [25], and there is little support for a regular cycle $[26,28,30]$. The nasal cycle is not only limited to the human nose, as it has been found in the rat and rabbit [31], the domestic pig [32, 33], the cat [34] and the dog [35], and appears to be a universal phenomenon at least in all mammals and possibly other animals.

We are not normally aware of the alternation in nasal airflow between the two nasal passages as the total nasal resistance remains relatively constant because of the reciprocal relationship between the two sides of the nose [36].

The changes in nasal airway resistance are under the control of the autonomic nerve supply to the blood vessels of the mucosa.

\section{Autonomic control of nasal venous sinusoids}

The spontaneous reciprocal changes in nasal airway resistance associated with the nasal cycle are caused by congestion and decongestion of the venous sinusoids lining the nasal mucosa. The nasal venous sinusoids form a spongy tissue, similar to erectile tissue, which is particularly well-developed at the anterior end of the nasal septum and the inferior turbinate [37-39].

The nasal venous sinusoids have a dense sympathetic adrenergic innervation, and there is evidence that the filling of the venous sinusoids and changes in nasal airway resistance are controlled by the sympathetic nerves to the nose. Electrical stimulation of the cervical sympathetic nerve causes a pronounced nasal vasoconstriction and a decrease in nasal airway resistance [32, 40-46]. The spontaneous reciprocal changes in nasal airway resistance, which constitute the nasal cycle, have been reported to be abolished after section of the cervical sympathetic nerve or local anaesthesia of the stellate ganglion [32, 47, 48]. In 1953, STOKSTEd [3] speculated that oscillations in sympathetic tone regulated the nasal cycle and that there was a nasal control centre in the hypothalamus. Experiments on anaesthetized cats have demonstrated that pronounced nasal vasoconstriction can be induced on electrical stimulation of localized areas of the hypothalamus [49]. Stimulation of the hypothalamus normally caused a bilateral vasoconstrictor response but in another series of experiments on the cat reciprocal changes in nasal vasomotor activity were induced by electrical stimulation of the brain stem [34], and this area of the brain which regulates respiration is a more likely site for a nasal control centre.

\section{The role of the nasal cycle in the production of nasal fluid}

The earliest theory on the generation of nasal fluid was that it was derived from the cranial cavity and slowly filtered through the cribriform plate into the nasal cavity. This concept prevailed until the discovery of glandular tissue in the respiratory mucosa and this tissue was then assumed to be the only source of nasal fluid [50]. The early reports on the origin of nasal fluid and its relationship to the nasal cycle, therefore, refer to nasal secretions rather than nasal fluid, and it is only relatively recently that the nasal fluid lining the nasal cavity has been understood to be a mixture of several elements, including secretions from glands and plasma exudate from nasal blood vessels. The close relationship between the generation of nasal fluid and the decongestion of the nasal venous sinusoids has been described independently by several eminent clinicians. 
In 1895, WRIGHT [51] reported that a mucous nasal secretion was associated with the decongestion phase of the nasal venous sinusoids but he ascribed this to a valve-like action of the veins at the mouth of the glands. HEETDERKS [2] (1927) carefully observed the vasomotor and secretory activity of the nasal mucosa in healthy volunteers and reported that the nasal secretions over the mucous membrane had a definite relation to the congestion of the turbinal structures. He stated that "in the nasal mucous membrane, however, there is an apparatus capable of furnishing water, namely, the erectile tissue of the turbinate bodies". This was not the first time that the production of nasal secretion had been linked to the nasal venous sinusoids, as LiLLIE [52] (1923) stated that "the mucous membrane of one nasal cavity is filling to a point approaching obstruction while the other is throwing off its secretion". A similar link between the vasomotor activity of the nasal venous sinusoids and secretion was also reported by BEICKERT [47] 1951, who stated "that in many normal cases the stronger secretion of the nasal mucous membranes coincides with the concha showing decreased swelling".

The early descriptions by LiLlie [52] (1923), HeEtDERKS [2] (1927) and BEICKERT [47] (1951) linking the formation of nasal fluid to the congestion and decongestion of nasal venous sinusoids appear to have been overlooked by later investigators, as the fenestrated nasal capillaries are implicated by CAUNA [39] (1982) as the source of plasma exudate involved in the humidification of inspired air. Studies on the generation of nasal plasma exudate associated with rhinitis or nasal challenge have, in general, explained the production of plasma exudate as being dependent on an increase in capillary permeability due to the actions of mediators of inflammation, and the nasal venous sinusoids have not been implicated as a source of plasma exudate [11, 53].

Interest in the nasal venous sinusoids as a source of nasal fluid has been reawakened by some elegant morphological studies by Grevers and Herrman [15, 17]. The morphological studies have demonstrated that there are fenestrated endothelial walls in the smooth muscle around the nasal venous sinusoids. The authors speculated that "these fenestrations, which are also located underneath the epithelium, might be even more important for secretory mechanisms of the nasal mucosa because of a much higher fluid capacity of these structures compared to vessels of the microcirculatory system" [17].

These observations on the role of the venous sinusoids in the production of nasal fluid, together with the anatomical evidence for endothelial fenestrations, implicate the nasal venous sinusoids in the generation of plasma exudate.

\section{Nasal venous sinusoids and plasma exudation}

The nasal venous sinusoids form a dense subepithelial plexus throughout the nasal mucosa and constitute a large reservoir of capacitance vessels, which contain a relatively much larger volume of blood than the nasal capillaries $[14,54]$. The presence of fenestration in these sinusoids and their polarization towards the epithelium supports a role in nasal fluid exchange similar to that which is generally accepted for the nasal capillaries [17].

One of the earliest reports that the nasal venous sinusoids could be involved in the generation of nasal fluid was made by WRIGHT [51] (1895), who accounted for the watery nasal secretion at the start of a nasal infection as "transudation directly from the blood vessels through the areolar tissue and the surface epithelium".

The periodic congestion and decongestion of the nasal venous sinusoids associated with the nasal cycle may contribute to plasma exudation and nasal fluid formation in two ways: firstly, by physically stretching the nasal epithelium; and, secondly, by increasing the hydrostatic pressure within the nasal venous sinusoids.

In the congestive phase of the nasal cycle, the venous sinusoids on one side of the nose are grossly distended, to such an extent that the anterior tip of the inferior turbinate may move forwards up to $5 \mathrm{~mm}$ [55]. This gross congestion of the nasal sinusoids will have two main effects, which may influence nasal fluid formation. Firstly, the distension of the venous sinusoids will physically stretch the smooth muscle around the sinusoids and the capillary plexus beneath the epithelium. The "unzipping" effect on cellular tight junctions and distension of fenestrations both in capillaries and veins is likely to increase permeability and facilitate plasma exudation onto the surface of the nasal epithelium. Secondly, congestion of the nasal venous sinusoids will be associated with an increased hydrostatic pressure within the sinusoids and this will facilitate the filtration of plasma and the formation of a plasma exudate. Relatively small distending pressures, as low as $5 \mathrm{cmH}_{2} \mathrm{O}$, have been shown to increase the permeability of tracheal epithelium and cause the transfer of macromolecules across the epithelium [10, 56, 57], and a similar increase in permeability is likely to occur across the nasal epithelium.

Those clinicians who have reported a relationship between the nasal cycle and nasal secretions (WRIGHT [51] (1895), Lillie [52] (1923), HeEtderks [2] (1927), BEICKERT [47] (1951)) have all commented that the nasal secretions are generated during the period of decongestion when the venous sinusoids are actively contracting under the influence of an increased sympathetic tone. During the period of decongestion, the blood held in the venous sinusoids will be subject to an increase in hydrostatic pressure due to the contraction of the smooth muscle surrounding the sinusoid and this could act to squeeze out plasma through the fenestrated endothelium towards the epithelium. Thus, the periodic congestion and decongestion of the nasal venous sinusoids associated with the nasal cycle may act as a "pump" or "filtration" mechanism to form a plasma exudate. The operation of an active pumping mechanism for the venous sinusoids, under the control of the sympathetic nervous system, is supported by the observation that the nasal mucosa is dry following ipsilateral interruption of the sympathetic nerve supply to the nose associated with Horners syndrome or stellate ganglion blockade [47]. Studies on the anaesthetized cat have shown that, on simultaneous electrical stimulation of parasympathetic and sympathetic 
nerves to the nose, a watery nasal fluid is formed during a pronounced vasoconstrictor response and that the fluid formation is reduced by ipsilateral sympathectomy [58].

The increased nasal fluid formation during the decongestion phase of the nasal cycle could be due to an oscillation in parasympathetic tone to the nasal glands in the same way that the sympathetic tone alternates from one side of the nose to the other. However, this seems unlikely, as there is no evidence to support an oscillation in parasympathetic tone to the nose, and the abolition of the nasal cycle on blockade of the sympathetic nerves to the nose also tends to eliminate the involvement of parasympathetic nerves in the periodic generation of nasal fluid associated with the nasal cycle. The glandular secretions appear to be mainly controlled by nasal reflexes, and physical or chemical irritation of nasal sensory nerves triggers sneezing and a profuse watery nasal fluid [36].

The various observations that nasal secretion is increased during the decongestion phase of the nasal cycle may, therefore, be explained by an increased formation of plasma exudate as the venous sinusoids contract and squeeze out a cell-free plasma filtrate. This does not mean that plasma exudation only occurs during the decongestion phase of the nasal cycle but it does indicate that there may be an increase in plasma exudation during this phase.

Experimental confirmation of a relationship between the nasal cycle and plasma exudation could be made by serial measurements of nasal airway resistance and nasal lavage to determine any correlation between nasal airway resistance (i.e. congestion/decongestion of venous sinusoids) and plasma protein content of nasal lavage.

The tracheobronchial venous sinusoids form a dense plexus beneath the respiratory epithelium of the lower respiratory tract and these sinusoids also become congested when the airway is inflamed. It is interesting to speculate that these blood vessels may also participate in the generation of plasma exudate, as has been proposed for the nasal venous sinusoids in this review.

\section{The role of nasal plasma exudate in respiratory defence}

The nose acts as the first line of defence for the respiratory system as it filters and conditions the 10-20,000 L of air that we breathe each day. The particulate matter continuously deposited in the nose exposes the nasal mucosa to a continuous threat of infection as the inspired air contains numerous pathogenic viruses, bacteria, yeasts and fungi. The nasal mucosa is the most commonly infected tissue in the body, with over 200 viruses causing the common cold syndrome. If one considers the nose as the primary site of infection for other common illnesses, such a influenza, measles, mumps, chicken pox and many other childhood infections, then the importance of the nose as a first line of defence against respiratory infection becomes clear. Mucociliary clearance of deposited particulate matter and the immunoglobulin and bacteriostatic components of glandular secretions provide important defences against infection but one should also consider plasma exudation as another vital line of defence.

The plasma exudate in the nose and lower respiratory tract has been proposed to constitute an important component of respiratory defence [11] rather than merely a side-effect of inflammation. The mechanism for movement of the plasma exudate across the epithelium has not been determined in detail but there is sufficient evidence to indicate that the epithelial cells may separate or "unzip" to allow the intracellular movement of plasma [12]. This flow of plasma through the epithelium and around epithelial cells would provide an excellent mechanism to flush out invading microorganisms, such as viruses and bacteria. As well as this physical cleansing mechanism, the plasma contains immunoglobulins which would bind to viruses and bacterial cell walls and aid their neutralization. Without a mechanism for plasma exudation, it is difficult to see how plasma immunoglobulins would interact with infectious agents outside the vascular system. Plasma proteins are involved in the generation of inflammatory mediators, such as bradykinin and the complement system, and these are important components of the inflammatory response and defence against infection.

Plasma exudation through the nasal epithelium and onto the surface of the nasal mucosa provides a first line of defence against respiratory infection, and it is not surprising that plasma exudation is increased by physical and chemical challenges to the nose as any disruption of the normal nasal epithelium will open the way for infection.

In conclusion, the hypothesis put forward in this review is that the nasal cycle has a role in respiratory defence. The term nasal cycle may be a misnomer, as there is little evidence to indicate a regular periodicity to the reciprocal changes in nasal airway resistance, which constitute the nasal cycle. However, it is firmly established in the literature that each nasal passage undergoes periods of congestion and decongestion related to the filling and emptying of venous sinusoids in the nasal mucosa.

The relationship between the state of congestion of the nasal venous sinusoids and the formation of nasal secretions has been reported on several occasions and as discussed above could represent an oscillation in parasympathetic tone to nasal glands. The involvement of the parasympathetic nerve supply in the generation of these "periodic secretions" is, however, unlikely, as the nasal cycle and associated secretions are abolished after section or blockade of the sympathetic nerves to the nose.

The periodic congestion and decongestion of the nasal venous sinusoids provides a mechanism for the formation of plasma exudate. During the congestion phase, the bulk flow of plasma from the nasal capillaries and venous sinusoids onto the nasal epithelium will be facilitated both by stretching of the nasal blood vessels and an increase in the hydrostatic pressure within the blood vessels. Active vasoconstriction of the venous sinusoids during the decongestion phase of the cycle will tend to squeeze out a plasma exudate and this may represent the nasal "secretions" described by several authors.

The nasal plasma exudate has been described as a first line of respiratory defence by PERSSON and co-workers 
[10-13]. Therefore, contribution of the nasal cycle to the generation of plasma exudate may be seen as a contribution to respiratory defence.

The increase in amplitude of the nasal cycle associated with nasal infection may increase the generation of plasma exudate by increasing the distension of the nasal epithelium and the hydrostatic filtration pressure within the nasal blood vessels.

The present review, therefore, proposes that the periodic congestion and decongestion of nasal venous sinusoids may provide a mechanism for the generation of plasma exudate and that this mechanism is an important component of respiratory defence.

\section{References}

1. Sen H. Observations on the alternate erectility of the nasal mucous membrane. Lancet 1901; i: 564.

2. Heetderks DL. Observations on the reaction of normal nasal mucous membrane. Am J Med Sci 1927; 174: 231-244.

3. Stoksted P. Rhinometric measurements for determination of the nasal cycle. Acta Otolaryngol (Stockh) 1953; 109 (Suppl.): 159-175.

4. Bende M, Barrow I, Heptonstall J, et al. Changes in human nasal mucosa during experimental coronavirus common colds. Acta Otolaryngol (Stockh) 1989; 107: 262-269.

5. Eccles R, Reilly M, Eccles KSJ. Changes in the amplitude of the nasal cycle associated with symptoms of acute respiratory tract infection. Acta Otolaryngol (Stockh) 1996; 115: (in press).

6. Proetz AW. In: Essays on the applied physiology of the nose. 2nd ed. Saint Louis, Annals Publishing Co., 1953.

7. Negus V. In: The comparative anatomy and physiology of the nose and paranasal sinuses. Edinburgh, E. \& S. Livingstone, 1958.

8. Matthew OP, Sant'Ambogio G (1988) eds. Respiratory function of the upper airway. In: Lenfant $\mathrm{C}$, ed. Lung Biology in Health and Disease. New York, Marcel Dekker, 1988; p. 645.

9. Cole P. In: The respiratory role of the upper airways: a selective clinical and pathological review. St. Louis, Mosby-Year Book, 1993.

10. Persson CGA, Erjefalt I, Gustafsson B, Luts A. Subepithelial hydrostatic pressure may regulate plasma exudation across the mucosa. Int Arch Allergy Immunol 1990; 92: 148-153.

11. Persson CGA, Erjefalt I, Alkner U, et al. Plasma exudation as a first line respiratory mucosal defence. Clin Exp Allergy 1991; 21: 17-24.

12. Persson CGA. Plasma exudation in the airways: mechanisms and function. Eur Respir J 1991; 4: 1268-1274.

13. Persson CGA. Mucosal exudation in respiratory defence: neural or non-neural control? Int Arch Allergy Immunol 1991; 94: 222-226.

14. Grevers G, Heinzmann U. Vascular corrosion casts of the nasal mucosa. Acta Otolaryngol (Stockh) 1989; 107: 254-261.

15. Grevers G, Herrman U. Fenestrated endothelia in vessels of the nasal mucosa. Arch Oto-Rhino-Laryngol 1987; 244: 55-60.

16. Grevers G. Morphological aspects of nasal blood vessels, in cells and tissues: a three dimensional approach by modern techniques in microscopy. New York, Alan R. Liss Inc, 1989; pp. 451-456.

17. Grevers G. The role of fenestrated vessels for the secretory process in the nasal mucosa: a histological and transmission electron microscopic study in the rabbit. Laryngoscope 1993; 103: 1255-1258.

18. Grevers G. Electron-microscopic observations on the muscular coat of swell bodies in human nasal mucosa. Laryngoscope 1994; 104: 1285-1289.

19. Grevers G, Kamargakis WN. Morphology of endonasal "cavernous" tissue with special reference to muscular pads or "cushions". Laryngorhinootologie 1994; 73: 573-576.

20. Grevers G, Kamargakis WN. Intravascular smooth muscle fibers and muscular bolsters in nasal swell bodies of humans. Ann Otol Rhinol Laryngol 1995; 104: 144-148.

21. Cole P, Haight JSJ. Posture and the nasal cycle. Ann Otol Rhinol Laryngol 1986; 95: 233-237.

22. Kayser R. Die exakte Messung der Luftdurchgängigkeit der Nase. Arch Laryngol Rhinol 1895; 3: 101-120.

23. Connell JT. Reciprocal nasal congestion/decongestion. Am Acad Ophthalmol Otolaryngol 1968; 72: 422-425.

24. Hasegawa M, Kern EB. The human nasal cycle. Mayo Clin Proc 1977; 52: 28-34.

25. Eccles R. The central rhythm of the nasal cycle. Acta Otolaryngol (Stockh) 1978; 86: 464-468.

26. Kern EB. The noncycle nose. Rhinology 1981; 19: 5974.

27. Cauwenberge PB v, Deleye L. Nasal cycle in children. Arch Otolaryngol 1984; 110: 108-110.

28. Gilbert AN, Rosenwasser AM. Biological rhythmicity of nasal airway patency: a re-examination of the nasal cycle. Acta Otolaryngol (Stockh) 1987; 104: 180-186.

29. Fox GP, Matthews TG. The nasal cycle in infants. Ir Med J 1991; 84: 24-25.

30. Juto JE, Lundberg C. Variation in nasal-mucosa congestion during rest. Acta Otolaryngol (Stockh) 1984; 98 : 136-139.

31. Bojsen-Moller F, Fahrenkrug J. Nasal swell bodies and cyclic changes in the air passages of the rat and rabbit nose. Anat 1971; 110: 25-37.

32. Eccles R. The domestic pig as an experimental animal for studies on the nasal cycle. Acta Otolaryngol (Stockh) 1978; 85: 431-436.

33. Campbell WM, Kern EB. The nasal cycle in swine. Rhinology 1981; 19: 127-148.

34. Bamford OS, Eccles R. The central reciprocal control of nasal vasomotor oscillations. Pflügers Arch 1982; 394: 139-143.

35. Webber RL, Jeffcoat MK, Harman JT, Ruttimann UE. Demonstration of the nasal cycle in the beagle dog. $J$ Comput Assist Tomogr 1987; 11: 869-871.

36. Eccles R. Neurological and pharmacological considerations. In: Proctor DF, Andersen I, eds. The Nose, Upper Airways Physiology and the Atmospheric Environment. Amsterdam, Elsevier, 1982; 191: pp. 214.

37. Burnham HH. An anatomical investigation of blood vessels of the lateral nasal wall and their relation to turbinates and sinuses. J Laryngol Otol 1935; 50: 569593.

38. Burnham HH. A clinical study of the inferior turbinate cavernous tissue; its divisions and their significance. Can Med Assoc J 1941; 44: 477-481.

39. Cauna N. Blood and nerve supply of the nasal lining. In: Proctor DF, Andersen I, eds. The Nose, Upper Airways Physiology and the Atmospheric Environment. Amsterdam, Elsevier, 1982; pp. 45-69. 
40. Tschalussow MA. Innervation der Gefässe der Nasenschleimhaut. Pflügers Arch 1913; 151: 523-542.

41. Blier Z. Physiology of the sphenopalatine ganglion. Am J Physiol 1930; 93: 398-406.

42. Malcolmson KG. The vasomotor activities of the nasal mucous membrane. J Laryngol Otol 1959; 37: 73-98.

43. Eccles R, Wilson H. The autonomic innervation of the nasal blood vessels of the cat. J Physiol (Lond) 1974; 238: 549-560.

44. Malm L. Stimulation of sympathetic nerve fibres to the nose in cats. Acta Otolaryngol (Stockh) 1973; 75: 519-526.

45. Anggard A, Edwall L. The effects of sympathetic nerve stimulation on the tracer disappearance rate and local blood content in the nasal mucosa of the cat. Acta Otolaryngol (Stockh) 1974; 77: 131-139.

46. Eccles R, Wallis DI. Characteristics of the sympathetic innervation of the nictitating membrane and of the vasculature of the nose and tongue of the cat. J Neural Transm 1976; 39: 13-130.

47. Beickert P. Halbseitenrhythmus der vegetativen Innervation. Arch Ohr-Hals-Heilk 1951; 157: 404-411.

48. Stoksted P, Thomsen K. Changes in the nasal cycle under stellate ganglion blockade. Acta Otolaryngol (Stockh) 1953; Suppl. 109: 176-181.

49. Eccles R, Lee RL. The influence of the hypothalamus on the sympathetic innervation of the nasal vasculature of the cat. Acta Otolaryngol (Stockh) 1981; 91: 127-134.

50. Schaeffer JP. The mucous membrane of the nasal cavity and the paranasal sinuses. In: Cowdry EV, ed. Special Cytology. The Form and Functions of the Cell in Health and Disease. New York, Paul B. Hoeber, 1932; pp. 107-129.

51. Wright JW. A consideration of the vascular mechanism of the nasal mucous membrane and its relations to certain pathological processes. Am J Med Sci 1895; 109: 516-523.

52. Lillie HI. Some practical considerations of the physiology of the upper respiratory tract. J Iowa Med Soc 1923; 13: 403-408.

53. Eccles R. Physiology of nasal secretion. Eur J Respir Dis 1983; 62: 115-119.

54. Malm L. Resistance and capacitance vessels in the nasal mucosa. Rhinology 1975; 13: 85-89.

55. Haight JSJ, Cole P. Site and function of the nasal valve. Laryngoscope 1983; 93: 49-55.

56. Gustafsson BG, Persson CGA. Asymmetrical effects of increases in hydrostatic pressure on macromolecular movement across the airway mucosa: a study in guinea-pig tracheal tube preparations. Clin Exp Allergy 1991; 21: 121-126.

57. Kondo M, Finkbeiner WE, Widdicombe JH. Changes in permeability of dog tracheal epithelium in response to hydrostatic pressure. Am J Physiol 1992; 262: L176-L182.

58. Wilson H, Yates MS. Sympathetic nerves and nasal secretion in the cat. Acta Otolaryngol (Stockh) 1978; 85: 426-430. 